\title{
EXTREME CONDITIONS IN A CLOSE ANALOG TO THE YOUNG SOLAR SYSTEM: HERSCHEL OBSERVATIONS OF $\epsilon$ ERIDANI
}

\author{
J. S. Greaves ${ }^{1}$, B. Sibthorpe ${ }^{2,3}$, B. Acke ${ }^{4}$, E. E. Pantin ${ }^{5}$, B. Vandenbussche ${ }^{4}$, G. Olofsson ${ }^{6}$, C. Dominik $^{7,8}$, M. J. Barlow ${ }^{9}$, \\ G. J. Bendo ${ }^{10}$, J. A. D. L. Blommaert ${ }^{4}, 11$, A. Brandeker ${ }^{6}$, B. L. DE VRIes ${ }^{6}$, W. R. F. Dent ${ }^{12}$, J. Di Francesco $^{13}$, \\ M. Fridlund ${ }^{14,15}$, W. K. Gear ${ }^{16}$, P. M. Harvey ${ }^{17}$, M. R. Hogerheijde ${ }^{18}$, W. S. Holland ${ }^{2,19}$, R. J. IVISON $^{19,20}$, \\ R. Liseau ${ }^{21}$, B. C. Matthews ${ }^{13,22}$, G. L. Pilbratt ${ }^{14}$, H. J. Walker ${ }^{23}$, and C. Waelkens ${ }^{4}$ \\ ${ }^{1}$ SUPA, Physics and Astronomy, University of St Andrews, North Haugh, St Andrews, Fife KY16 9SS, UK; jsg5@ st-andrews.ac.uk \\ ${ }^{2}$ UK Astronomy Technology Centre, Royal Observatory Edinburgh, Blackford Hill, Edinburgh EH9 3HJ, UK \\ ${ }^{3}$ SRON, Sorbonnelaan 2, 3584-CA Utrecht, The Netherlands \\ ${ }^{4}$ Instituut voor Sterrenkunde, K. U. Leuven, Celestijnenlaan 200D, B-3001 Leuven, Belgium \\ ${ }^{5}$ Laboratoire AIM, CEA/DSM-CNRS-Universit Paris Diderot, IRFU/Service dAstrophysique, Bat.709, CEA-Saclay, F-91191 Gif- sur-Yvette Cedex, France \\ ${ }^{6}$ Stockholm University Astrobiology Centre, SE-106 91 Stockholm, Sweden \\ ${ }^{7}$ Astronomical Institute Anton Pannekoek, University of Amsterdam, P.O. Box 94249, 1090-GE Amsterdam, The Netherlands \\ ${ }^{8}$ Department of Astrophysics/IMAPP, Radboud University Nijmegen, P.O. Box 9010, 6500-GL Nijmegen, The Netherlands \\ ${ }^{9}$ Department of Physics and Astronomy, University College London, Gower Street, London WC1E 6BT, UK \\ ${ }_{10}$ Jodrell Bank Centre for Astrophysics, School of Physics and Astronomy, University of Manchester, Oxford Road, Manchester M13 9PL, UK \\ ${ }^{11}$ Astronomy and Astrophysics Research Group, Department of Physics and Astrophysics, V. U. Brussel, Pleinlaan 2, B-1050 Brussels, Belgium \\ 12 ALMA SCO, Alonso de Cordova 3107, Vitacura, Casilla 763 0355, Santiago, Chile \\ ${ }^{13}$ Herzberg Astronomy and Astrophysics Programs, National Research Council of Canada, 5071 West Saanich Road, Victoria, BC, V9E 2E7, Canada \\ ${ }^{14}$ ESA, SRE-SA, Keplerlaan 1, NL-2201 AZ Noordwijk, The Netherlands \\ ${ }^{15}$ Institute of planetary Research, German Aerospace Center, Rutherfordstrasse 2, D-12489 Berlin, Germany \\ ${ }^{16}$ School of Physics and Astronomy, Cardiff University, Queens Buildings, The Parade, Cardiff CF24 3AA, UK \\ ${ }^{17}$ Astronomy Department, University of Texas, Austin, TX 78712, USA \\ ${ }^{18}$ Leiden Observatory, Leiden University, P.O. Box 9513, 2300-RA Leiden, The Netherlands \\ ${ }^{19}$ Institute for Astronomy, University of Edinburgh, Royal Observatory, Blackford Hill, Edinburgh EH9 3HJ, UK \\ ${ }^{21}$ Earth and Space Sciences, Chalmers University of Technology, Onsala Space Observatory, SE-439 92 Onsala, Sweden \\ ${ }^{22}$ University of Victoria, Finnerty Road, Victoria, BC, V8W 3P6, Canada \\ ${ }^{23}$ RAL Space, Science and Technology Facilities Council, Rutherford Appleton Laboratory, Chilton, Didcot, Oxon OX11 0QX, UK \\ Received 2014 April 24; accepted 2014 July 7; published 2014 July 25
}

\begin{abstract}
Far-infrared Herschel images of the $\epsilon$ Eridani system, seen at a fifth of the Sun's present age, resolve two belts of debris emission. Fits to the $160 \mu \mathrm{m}$ PACS image yield radial spans for these belts of 12-16 and 54-68 AU. The south end of the outer belt is $\approx 10 \%$ brighter than the north end in the PACS+SPIRE images at 160, 250, and $350 \mu \mathrm{m}$, indicating a pericenter glow attributable to a planet "c." From this asymmetry and an upper bound on the offset of the belt center, this second planet should be mildly eccentric $\left(e_{c} \approx 0.03-0.3\right)$. Compared to the asteroid and Kuiper Belts of the young Sun, the $\epsilon$ Eri belts are intermediate in brightness and more similar to each other, with up to $20 \mathrm{~km}$ sized collisional fragments in the inner belt totaling $\approx 5 \%$ of an Earth mass. This reservoir may feed the hot dust close to the star and could send many impactors through the Habitable Zone, especially if it is being perturbed by the suspected planet $\epsilon$ Eri b, at semi-major axis $\approx 3 \mathrm{AU}$.
\end{abstract}

Key words: circumstellar matter - planet-disk interactions - stars: individual ( $\epsilon$ Eridani)

Online-only material: color figures

\section{INTRODUCTION}

The $\epsilon$ Eridani system is the archetype of a debris disk around a Sun-like star, discovered nearly three decades ago with IRAS (Aumann 1985). At only $3.2 \mathrm{pc}$ distance, the star is resolved by optical interferometry (e.g., Baines \& Armstrong 2012) establishing it at around 0.32, 0.74, and 0.82 times the Sun's luminosity, radius, and mass, respectively, and with an age fitted at $850 \mathrm{Myr}$ (Di Folco et al. 2004). A Jovian planet is suspected to orbit at around $3 \mathrm{AU}$ from $\epsilon$ Eri, from radial velocity wobble and Hubble Space Telescope/Hipparcos astrometry, but a consistent planetary solution is elusive and complicated by a possible $7 \mathrm{yr}$ modulation of the stellar activity cycle (Metcalfe et al. 2013; Anglada-Escudé \& Butler 2012; Reffert \& Quirrenbach 2011; Benedict et al. 2006).

Far-infrared observations with Spitzer (Backman et al. 2009) indicated two unresolved warm inner dust rings, as well as a wider icy outer belt, confirmed by $350 \mu \mathrm{m}$ Caltech Submil- limeter Observatory (CSO) imaging. While the warmest dust may trickle in past the candidate planet (Reidemeister et al. 2011; Brogi et al. 2009), there is no hot component very near the star detectable with near-infrared interferometry (Di Folco et al. 2007). The cold outer debris belt was earlier resolved by SCUBA at 450 and $850 \mu \mathrm{m}$, including detection of clumps suspected to be the locations of rotational resonance with an outer planet (Greaves et al. 2005, 1998, with follow-up detections with SCUBA-2; J. S. Greaves et al. in preparation). Direct imaging searches for this planet have set upper limits of a few Jupiter masses (Marengo et al. 2009; Heinze et al. 2008; Janson et al. 2008).

The similarities of $\epsilon$ Eri to inferences about the young solar system (e.g., Booth et al. 2009) make it worth assessing prospects for a terrestrial planet in the Habitable Zone spanning 0.5-1 AU (Baines \& Armstrong 2012), especially as we see the system at about a fifth of the Sun's current age. This is roughly when the solar system is thought to have undergone shifting of 

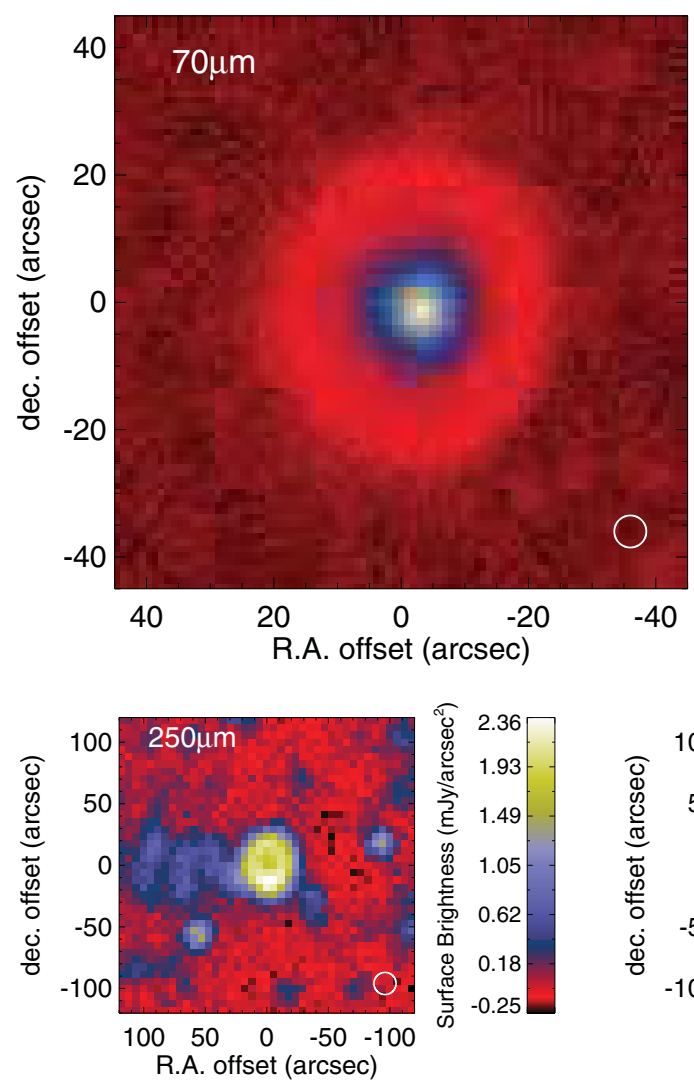
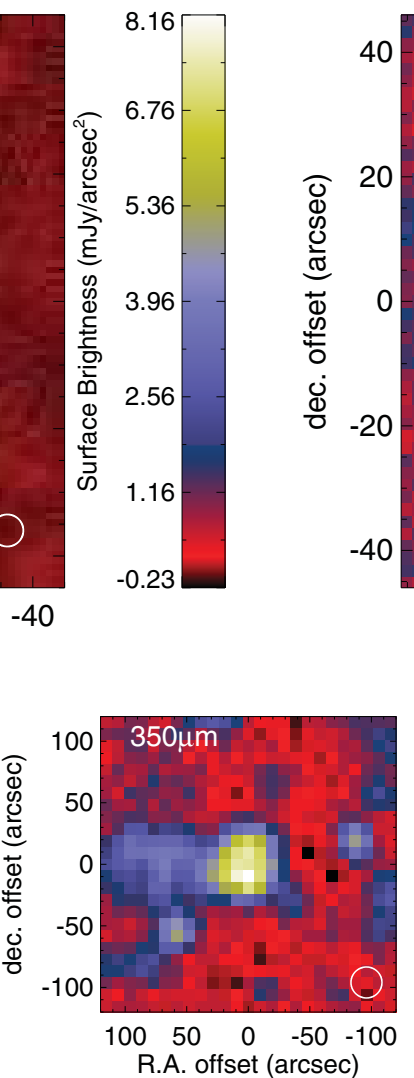
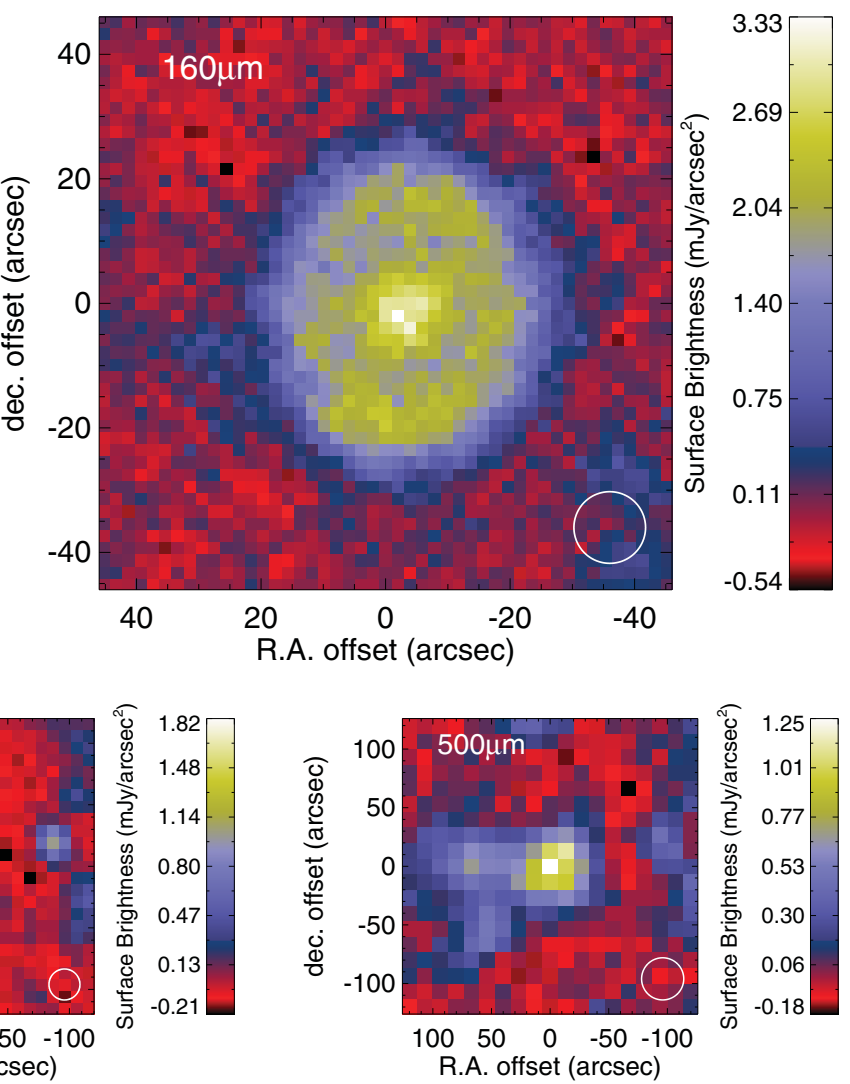

Figure 1. Top row: PACS images toward $\epsilon$ Eri plotted in linear false-color scales for surface brightness and with pixel sizes of $1^{\prime \prime}$ and $2^{\prime \prime}$ at 70 and $160 \mu \mathrm{m}$, respectively. Bottom row: SPIRE images at 250,350,500 $\mu$ m with pixels of $6^{\prime \prime}, 10^{\prime \prime}, 14^{\prime \prime}$, respectively. Larger fields are shown in these panels to demonstrate the presence of presumed dusty galaxies, most prominently at positive-R.A. offsets.

(A color version of this figure is available in the online journal.)

giant planet orbits with radical perturbations of the comet and asteroid belts (Walsh et al. 2011; Gomes et al. 2005). Here, we examine the two debris environments and compare their sources of potential planetary impactors.

\section{OBSERVATIONS WITH HERSCHEL}

The Herschel ${ }^{24}$ Guaranteed Time Key Project "Stellar Disk Evolution" (PI: G. Olofsson) obtained simultaneous PACS 70/ $160 \mu \mathrm{m}$ images on 2011 March 5 (observation IDs 1342216123/ 4), using two cross-scans at the medium scan speed of $20^{\prime \prime} \mathrm{s}^{-1}$. The SPIRE 250, 350, and $500 \mu \mathrm{m}$ images are from one observation made on 2010 February 25 (ID 1342191177), using Large Map mode at $30^{\prime \prime} \mathrm{s}^{-1}$ scan rate. Observation durations were $5478 \times 2$ and $2923 \mathrm{~s}$, covering fields of approximately $7^{\prime}$ and $12^{\prime}$, with PACS and SPIRE, respectively. Detailed descriptions of Herschel and the PACS and SPIRE instruments are presented by Pilbratt et al. (2010), Poglitsch et al. (2010), and Griffin et al. (2010). The data were processed in the Herschel interactive processing environment, following procedures as established for Fomalhaut (Acke et al. 2012).

The pixel scales used in the images at 70, 160, 250, 350, and $500 \mu \mathrm{m}$ are $1^{\prime \prime}, 2^{\prime \prime}, 6^{\prime \prime}, 10^{\prime \prime}$, and $14^{\prime \prime}$, respectively, while mean FWHM of the point-spread functions (PSFs) are typically 5".6, 11".4, 18.'2, 24."9, and 36."3 (18-117 AU). The absolute co-ordinate frame was checked for the PACS data using three

\footnotetext{
${ }^{24}$ Herschel is an ESA space observatory with science instruments provided by European-led Principal Investigator consortia and with important participation from NASA.
}

Two Micron All Sky Survey sources that appear in the field. At $70 \mu \mathrm{m}$, these are on average within $1^{\prime \prime}(1$ pixel) of their nominal positions (worst case: 1.5 pixels, scatter on each axis: 0.5 pixels) but they give too little information for any re-registering of the images. Another minor aspect of image fidelity is that the PSF has low-level tri-lobe structure due to the secondary mirror's support structure (see the report "bolopsf_20.pdf" at the PACS calibration Web site), and this contributes to the shape of the central peak at $70 \mu \mathrm{m}$.

\section{IMAGING RESULTS}

The five Herschel images toward $\epsilon$ Eri are shown in Figure 1 . The central star is brightest at the shortest wavelengths, becoming negligible by the submillimeter bands, where the outer dust belt dominates. The overall structure is very similar to ground-based submillimeter images, such as the $350 \mu \mathrm{m}$ data of Backman et al. (2009). Background objects are particularly prominent in the submillimeter on the eastern (positive-R.A.) side of the system. The proper motion of the star-approximately $1^{\prime \prime}$ to lower right ascension (R.A.) per year-has moved it westward over these structures on decadal timescales. This contaminant explains the more east-west extension seen in the earliest images (Greaves et al. 1998) - hence, there is overall agreement on a slightly inclined disk with its apparent major axis lying close to north-south.

The warm belt within a few arcseconds of the star is resolved here for the first time, most noticeably at $70 \mu \mathrm{m}$ (see the radial profiles in Figure 2). By $160 \mu \mathrm{m}$, the cool outer belt is dominant (Figure 3), and there is a clear gap (Figure 1) between the 


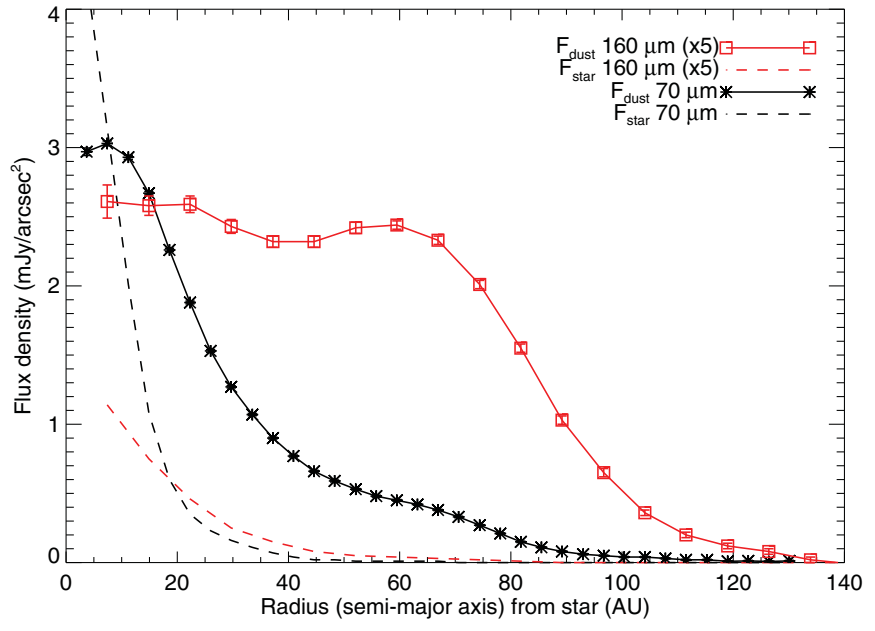

Figure 2. Radial flux profiles from the $70,160 \mu \mathrm{m}$ PACS images. Photospheresubtracted disk-profile points are mean signals within elliptical annuli (correcting for $30^{\circ}$ inclination from the sky plane), with their standard errors, plotted against de-projected radius. Thin curves show the flux profile for the star, with photospheric signals (Backman et al. 2009) adapted for the PACS passbands. The errorbars show standard errors for the number of independent pixels per annulus $(\approx N$ (pixels) $/ 6$ for 1 pixel width and PSFs $\approx 6$ pixels across at half-maximum $)$, so adjacent annuli are not independent. Standard deviations were derived from the signal dispersion in 1 pixel wide strips across the image background.

(A color version of this figure is available in the online journal.)

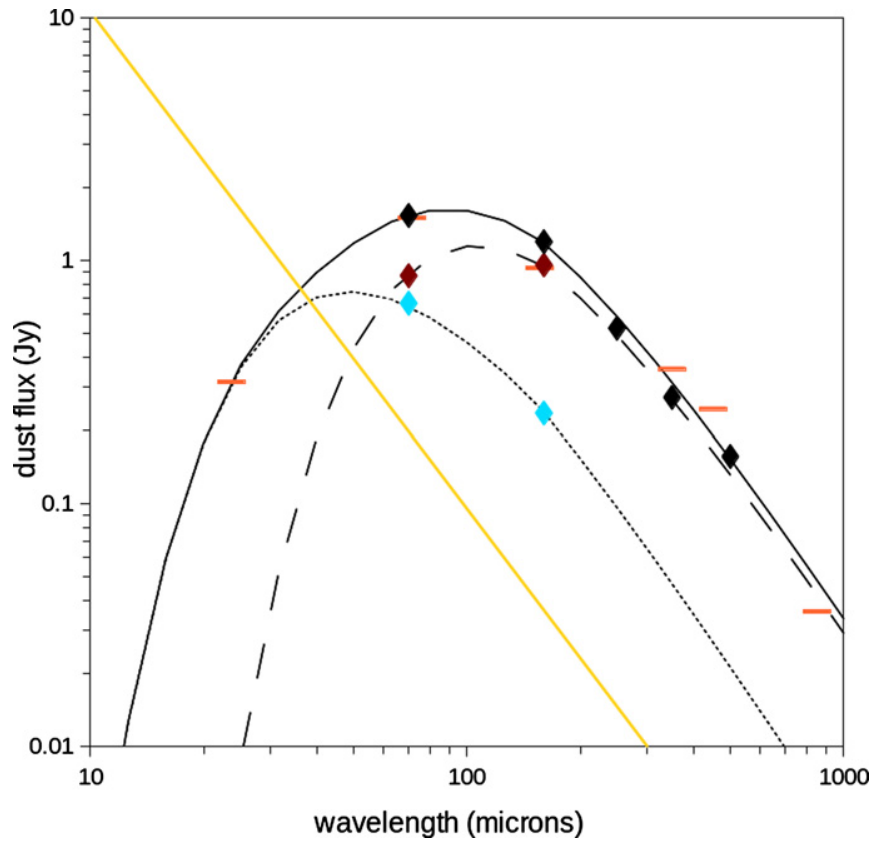

Figure 3. Dust fluxes around $\epsilon$ Eri in the mid-infrared to submillimeter; photospheric signals have been subtracted (Backman et al. 2009). Black diamonds are Herschel data (with the inner and outer rings shown separately in light blue and dark red, respectively), orange bars are prior measurements from Table 1, and the yellow line is the photospheric signal. The black curves show blackbody emission at $105 \mathrm{~K}$ (dotted) and $48 \mathrm{~K}$ (dashed), both modified by a declining emissivity of index 0.4 longward of $150 \mu \mathrm{m}$, and summed to form the solid black line.

(A color version of this figure is available in the online journal.)

two zones. The SPIRE images are dominated by the outer belt, with central clearing visible at both 250 and $350 \mu \mathrm{m}$ (Figure 1). Backman et al. (2009) suggested there is a "halo" of small grains at 90-110 AU, and the radial tail of emission seen by PACS out to at least $125 \mathrm{AU}$ (Figure 2) agrees with their $350 \mu \mathrm{m}$ data. Dust fluxes are listed in Table 1, including the two components separately where resolved by PACS.
Table 1

Debris Excess Fluxes above the Photosphere

\begin{tabular}{lcc}
\hline \hline $\begin{array}{l}\text { Wavelength } \\
(\mu \mathrm{m})\end{array}$ & $\begin{array}{c}\text { Dust Flux } \\
(\mathrm{Jy})\end{array}$ & Notes \\
\hline \multicolumn{3}{c}{ Herschel data } \\
\hline 70 & $0.67 \pm 0.01$ & Inner ring, PACS \\
70 & $0.87 \pm 0.01$ & Outer ring, PACS \\
160 & $0.24 \pm 0.02$ & Inner ring, PACS \\
160 & $0.96 \pm 0.03$ & Outer ring, PACS \\
250 & $0.51 \pm 0.03$ & SPIRE \\
350 & $0.26 \pm 0.02$ & SPIRE \\
500 & $0.16 \pm 0.01$ & SPIRE \\
\hline & Previous results & \\
\hline 24 & $0.32 \pm 0.04$ & Spitzer $/$ MIPS \\
71 & $1.50 \pm 0.21$ & Spitzer $/$ MIPS \\
156 & $0.93 \pm 0.19$ & Spitzer $/$ MIPS \\
350 & $0.36 \pm 0.05$ & CSO/SHARC II \\
450 & $0.25 \pm 0.02$ & JCMT/SCUBA \\
850 & $0.036 \pm 0.003$ & JCMT/SCUBA \\
\hline
\end{tabular}

Notes. Previous results are from Backman et al. (2009) and Greaves et al. (2005). For SPIRE, errors are dominated by background estimation in the photometry, for an aperture of radius $40^{\prime \prime}$, and annuli $30^{\prime \prime}$ wide with inner edges at $60^{\prime \prime}$ or $150^{\prime \prime}$ and with/without blanking of the positive-R.A. features. For PACS, inner and outer belts were separated by fitting Gaussians to the radial profiles (Figure 2); errors are estimated from aperture photometry variations among background areas, of radii $20^{\prime \prime}$ and $40^{\prime \prime}$, respectively. Absolute flux calibration for Herschel contributes up to $5 \%$ additional uncertainty.

At $160 \mu \mathrm{m}$, the outer belt appears structured, but the most prominent brightening (to the north and south) is attributable to limb effects, where longer columns are viewed down the ends of the inclined ring. An additional north-to-south asymmetry is discussed below. There are also two slightly brightened patches at the east and west sides of the ring, but tests made by artificially moving the system around within the map showed that these can be produced solely by background fluctuations.

\subsection{Model Fits}

The two debris belts in the PACS images were fitted with model rings, fitting both wavelengths simultaneously. A minimal approach was used, adopting two uniform, circular, smoothdensity rings, and optimizing the overall fit with an "amoeba" multi-dimensional minimization algorithm. The disk radii, width, opening angle, and inclination are all free parameters, but the last two are common to both rings. In the data, the dust belts appear well centered on the star, but at $160 \mu \mathrm{m}$ offsets of up to 1 pixel $\left(2^{\prime \prime}\right)$ were allowed for optimization. The common inclination was fitted as $32^{\circ}$ (from $160 \mu \mathrm{m}$, where the PSF is the most stable), and for comparison, a simplified flat ring fitted to the outer belt alone yielded $30^{\circ}$. This parameter thus appears robust to different models; a conservative upper bound on angle uncertainties is $\approx 5^{\circ}$ (see discussion below).

The fitting results show that the debris belts are quite radially confined $(\Delta R / R$ around $0.2-0.3)$ and separated by a substantial gap. The best-fit parameters for the $160 \mu \mathrm{m}$ image are two belts spanning 12-16 and 54-68 AU, with opening angles of $\pm 20^{\circ}$ and $\pm 11^{\circ}$, respectively. This indicates that both belts are quite vertically extended, with the particles in the inner one orbiting at greater angles with respect to the mid-plane. 
The radial and vertical extents are all fitted as being fractionally greater at $70 \mu \mathrm{m}$ than at $160 \mu \mathrm{m}$. The best fit gives radial spans of 3-21 and 36-72 AU, and opening angles of $\pm 23^{\circ}$ and $\pm 15^{\circ}$, respectively. This result may be attributable to emission from smaller particles being more dynamically dispersed, as in the models by Müller et al. (2010) of grains on eccentric orbits. These models were for the Vega system, for which Sibthorpe et al. (2010) also found larger disk radii at shorter Herschel wavelengths. In the models, higher particle eccentricities can lead to a "pile-up" of smaller grain sizes, plus enhanced collisions near the planetesimal belt, produce broader radial profiles of emission.

Backman et al. (2009) inferred the smaller ring to lie at $\sim 20$ AU from unresolved Spitzer data at 24-156 $\mu \mathrm{m}$, and the Herschel results are in good agreement with this, but placing it slightly inward at improved resolution. The innermost ring, which Backman et al. inferred to lie at around 3 AU from midinfrared excesses, is still below resolvable scales.

\subsection{Multiple Belts}

Many debris systems are now being found to have both warm and cold dust. However, when the emission is unresolved it is not clear whether this effect arises from two belts, a broad single belt, or grains with a range of properties (Booth et al. 2013; G. Kennedy et al., in preparation). Amongst the archetypes, Su et al. (2013) discuss Vega and Fomalhaut, both of which have warm excesses shortward of $30 \mu \mathrm{m}$, as well as large cold debris belts and hot dust near the star. The new Herschel images have confirmed the presence of two belts around $\epsilon$ Eri, and resolve the smaller one as centered approximately $14 \mathrm{AU}$ from the star, which is similar to inferred locations $\approx 8-14$ AU in the Vega and Fomalhaut systems.

However, $\epsilon$ Eri (spectral type K2 V) is much less luminous than the two A stars, so their middle belts are warmer- $\mathrm{Su}$ et al. (2013) find $\approx 170 \mathrm{~K}$, versus $105 \mathrm{~K}$ here (Figure 3)-and so the former may be better "asteroid belt" analogs. The Sun is comparable in luminosity to $\epsilon$ Eri but the asteroid belt is closer in, so these are again probably dissimilar in composition (Minton \& Malhotra 2010). However, there are marked structural similarities, as both $\epsilon$ Eri and the Sun have two planetesimal belts plus particles thought to have drifted inward (Reidemeister et al. 2011), forming the zodiacal belt in the solar case.

\subsection{Planetary Effects}

Resonant clumps have not been seen by Herschel around $\epsilon$ Eri, probably because emission drops off at wavelengths much larger than particle size, and the larger millimeter-emitters are much more readily trapped into resonances (Wyatt 2008). However, there is some azimuthal structure around the outer belt. A south-north flux asymmetry is seen in the 160, 250, and $350 \mu \mathrm{m}$ images, in addition to the limb-brightening effect noted above. The ratios of ( $\left.F_{\text {south }}: F_{\text {north }}\right)$ obtained for pixel pairs at 160,250 , and $350 \mu \mathrm{m}$, respectively, are 1.09, 1.12, and 1.11, with flux differences at $3.0,3.7$, and 2.5 times the pixel-topixel scatter (estimated within $3 \times 3$ boxes around each point). This asymmetry is robust as it has been identified with two independent instruments. The effect is typical of "pericenter glow, which is a forced shift of planetesimal orbits due to interactions with a planet, leading to warmer particles on the side of the belt that is closer to the star (Wyatt et al. 1999).

These contrast values seen for $\epsilon$ Eri are similar to measurements for the Fomalhaut debris disk at $160 \mu \mathrm{m}$ (Acke et al.
Table 2

Derived Properties for the $\epsilon$ Eri Belts

\begin{tabular}{lccccc}
\hline \hline Property & \multicolumn{2}{c}{$\epsilon$ Eri Belts } & & \multicolumn{2}{c}{ Young-Sun Belts } \\
\cline { 2 - 3 } & Inner & Outer & & Asteroid & Kuiper \\
\hline Mean radius (AU) & 14 & 61 & & 3 & 26 \\
Radial width (AU) & 4 & 14 & & 1 & 15 \\
Mean eccentricity & 0.17 & 0.10 & & $\sim 0.15$ & $\sim 0.05$ \\
$L_{\text {dust }} / L_{\text {star }}\left(10^{-5}\right)$ & 3 & 4 & & $\sim 0.5$ & $\sim 30$ \\
$D_{\text {br }}(\mathrm{km})$ & 20 & 2 & & $\sim 50$ & $\sim 8$ \\
$M\left(D \leqslant D_{\text {br }}\right)\left(M_{\oplus}\right)$ & 0.05 & 0.4 & & $\sim 0.001$ & $\sim 1.5$ \\
\hline
\end{tabular}

Notes. Properties are compared to those of the Sun's debris belts at a similar age, using model masses (Booth et al. 2009; Walsh et al. 2011) to derive f-values ( $L_{\text {dust }} / L_{\text {star }}$; Wyatt 2008). For the $\epsilon$ Eri belts, the calculations are in reverse, i.e., $f$ is used to infer masses. $D_{\text {br }}$ and $M\left(D \leqslant D_{\text {br }}\right)$ are from Equations (1) and (2), assuming a canonical size distribution $d N / d D \propto D^{-3.5}$ extending up to planetesimal diameters $D_{\max } \sim 2000 \mathrm{~km}$ as in the solar system, but with $f$ arising from bodies with $D<D_{\text {br }}$ only.

2012). A forced offset of the center of the belt from the star is also expected, but it is too small to identify for $\epsilon$ Eri, with the $160 \mu \mathrm{m}$ centroid lying within $2^{\prime \prime}$ (6.4 AU) of the star. The expected shift due to this forced eccentricity is $R_{\text {belt }} e_{\text {forced }}$ (Wyatt et al. 1999), so $e_{\text {forced }}$ is here $\lesssim 0.1$, compared to $e_{\text {forced }}$ of 0.12 due to Fomalhaut b (Acke et al. 2012). The level of brightness asymmetry depends on the (unknown) orientation at which we view the forced offset, but calculations by Wyatt et al. (1999) suggest $e_{\text {forced }} \gtrsim 0.03$ here. For these bounds, and assuming that the forcing is due to a planet "c" in the inter-belt gap at 16-54 $\mathrm{AU}$ (not to a $3 \mathrm{AU}$ companion), secular perturbation theory indicates a planetary eccentricity $e_{c} \approx 0.03-0.3$ (Wyatt et al. 1999). These calculations do not give any mass constraint, but upper limits from direct imaging searches suggest $\epsilon$ Eri c should not exceed about 5 Jupiter masses (Marengo et al. 2009).

\section{DISCUSSION: $\epsilon$ ERI AND THE YOUNG SUN}

The fractional luminosities of the two belts, $f=L_{\text {dust }} / L_{\text {star }}$ (Table 2), are now well established with the inclusion of the new data. The dust luminosities agree with the values of Backman et al. (2009) within $15 \%$, for grain temperatures differing by $\sim 5 \mathrm{~K}$ (Figure 3). Resolving the two belts has thus verified the earlier spectral energy distribution fitting, but with the advantage of refined values of the radial extents plus estimates of the opening angles. From the latter, mean eccentricities of the particles can also be derived, by setting them equal to the mean inclination (half the angle of opening from the mid-plane; e.g., Wyatt 2008). This is not necessarily to be expected, if the belts are sculpted by planets which can force planetesimal orbits, trap them into resonances, etc. However, for our comparison to the solar system, we note that the median $e: i$ ratio for Kuiper Belt Objects (KBOs) today (Murray-Clay \& Schlichting 2011, for discussion) is in fact close to equipartition.

The $\epsilon$ Eri system, at an age of around $850 \mathrm{Myr}$, can then be compared to models of the solar system before it was largely cleared in the "late heavy bombardment" (LHB) event. The timing of an LHB-analog event will depend on the planetary system architecture, but we assume here that such an event (1) is possible given the presence of planets, and (2) has not yet occurred because the planetesimal populations are still large.

We estimate belt masses including bodies up to the largest sizes that can have had a collisional break-up at the age of the 
system, $D_{\mathrm{br}}$. The summed mass in this collisional cascade is then given by (Wyatt 2008)

$$
M=2.7 L_{\text {dust }} / L_{\text {star }} R^{2} D_{\mathrm{bl}}^{0.5} D_{\mathrm{br}}^{0.5}
$$

for particles down to size $D_{\mathrm{bl}}$ that are blown out by radiation pressure ( 0.5 and $1 \mu \mathrm{m}$ for $\epsilon$ Eri and the Sun, respectively). $D_{\mathrm{br}}$ is derived from

$$
t=\left(5.210^{-10} / f\right) R^{4 / 3} \Delta R e^{-5 / 3} M_{*}^{-4 / 3} D_{\mathrm{bl}}^{-0.5} D_{\mathrm{br}}^{0.5} Q_{D \mathrm{br}}^{* 5 / 6},
$$

with mass $M_{*}$ of $0.85 M_{\odot}$ for $\epsilon$ Eri, and canonical values for planetesimal strength $Q_{D}^{*}$ from Wyatt et al. (2011). We caution that factors of a few are unlikely to be significant in the comparison of the two systems. For example, the $\epsilon$ Eri eccentricities would change by $30 \%-50 \%$ if the $70 \mu \mathrm{m}$ fit results were adopted instead of the $160 \mu \mathrm{m}$ values, and this would alter $D_{\text {br }}$ by factors of 2-4 for a fixed $t$. Also, the population size distribution was implicitly a power law of slope -3.5 above, but more realistically (Gáspár et al. 2012 and references therein), slopes may be around -3.65 to -3.8 . Pushing this to an extreme case of slope -4 (as seen for the largest, unevolved, KBOs), $t$ is $\propto D_{\mathrm{br}}^{2.5}$, and so if, e.g., $t$ is $30 \%$ uncertain, the break-up size is only known to a factor of two.

The results in Table 2 show many similarities between the double-belt systems of $\epsilon$ Eri and the Sun at that age. The main differences, to an external observer, would be the larger scales of the $\epsilon$ Eri belts, and their luminosities that are roughly equal, and intermediate to those of the Sun's belts. An implication is that the bodies inferred to be breaking up in the inner system of $\epsilon$ Eri, while of a tens-of-km class akin to the young asteroid belt, come from a mass reservoir that is around 50 times greater. In contrast, the outer belt is within a factor of a few of the mass of the pre-LHB Kuiper Belt in up to few-kilometer-size bodies.

\section{CONCLUSIONS}

The $\epsilon$ Eridani system has been shown to be moderately analogous to the solar system, when at a comparable age of a few hundred million years. However, the differences in the debris belts are rather greater than those between the stars. Most strikingly, the middle dust belt of $\epsilon$ Eri is inferred to be more massive by two orders of magnitude in bodies up to tens-of-kilometers class. The Sun's asteroids are perturbed by Jupiter, whereas the $\epsilon$ Eri belt at around $14 \mathrm{AU}$ is more dynamically separated from either planet candidate "b" or "c," so this may allow it to sustain a larger planetesimal population. As dust is thought to trickle in to around $3 \mathrm{AU}$ from $\epsilon$ Eri (Reidemeister et al. 2011), if parent bodies follow similar trajectories they could fall in further and might severely impact any planet in the Habitable Zone of $\epsilon$ Eri. Numerical simulations are important to assess the potential habitability of the system, especially as we see it at a time around that of the earliest known life on Earth.
We thank the referee for some very useful suggestions about the dynamics. PACS has been developed by a consortium of institutes led by MPE (Germany) and including UVIE (Austria); KU Leuven, CSL, IMEC (Belgium); CEA, LAM (France); MPIA (Germany); INAF-IFSI/OAA/OAP/ OAT, LENS, SISSA (Italy); IAC (Spain). This development has been supported by the funding agencies BMVIT (Austria), ESA-PRODEX (Belgium), CEA/CNES (France), DLR (Germany), ASI/INAF (Italy), and CICYT/MCYT (Spain). SPIRE has been developed by a consortium of institutes led by Cardiff University (UK) and including University of Lethbridge (Canada); NAOC (China); CEA, LAM (France); IFSI, Univ. Padua (Italy); IAC (Spain); Stockholm Observatory (Sweden); Imperial College London, RAL, UCL-MSSL, UKATC, University of Sussex (UK); and Caltech, JPL, NHSC, University of Colorado (USA). This development has been supported by national funding agencies: CSA (Canada); NAOC (China); CEA, CNES, CNRS (France); ASI (Italy); MCINN (Spain); SNSB (Sweden); STFC (UK); and NASA (USA).

\section{Facility: Herschel (PACS, SPIRE)}

\section{REFERENCES}

Acke, B., Min, M., Dominik, C., et al. 2102, A\&A, 540, 125 Anglada-Escudé, G., \& Butler, R. P. 2012, ApJS, 200, 15 Aumann, H. H. 1985, PASP, 97, 885

Backman, D., Marengo, M., Stapelfeldt, K., et al. 2009, ApJ, 690, 1522 Baines, E. K., \& Armstrong, J. T. 2012, ApJ, 744, 138

Benedict, G. F., McArthur, B. E., Gatewood, G., et al. 2006, AJ, 132, 2206 Booth, M., Kennedy, G., Sibthorpe, B., et al. 2013, MNRAS, 428, 1263

Booth, M., Wyatt, M. C., Morbidelli, A., Moro-Martin, A., \& Levison, H. F. 2009, MNRAS, 399, 385

Brogi, M., Marzari, F., \& Paolicchi, P. 2009, A\&A, 499, L13

Di Folco, E., Absil, O., Augereau, J.-C., et al. 2007, A\&A, 475, 243 Di Folco, E., Thévenin, F., Kervella, P., et al. 2004, A\&A, 426, 601

Gáspár, A., Psaltis, D., Rieke, G. H., \& Özel, F. 2012, ApJ, 754, 74

Gomes, R., Levison, H. F., Tsiganis, K., \& Morbidelli, A. 2005, Natur, 435, 466

Greaves, J. S., Holland, W. S., Moriarty-Schieven, G., et al. 1998, ApJL, 506, L133

Greaves, J. S., Holland, W. S., Wyatt, M. C., et al. 2005, ApJL, 619, L187

Griffin, M. J., Abergel, A., Abreu, A., et al. 2010, A\&A, 518, L2

Heinze, A. N., Hinz, P. M., Kenworthy, M., Miller, D., \& Sivanandam, S. 2008, ApJ, 688, 583

Janson, M., Apai, D., Zechmeister, M., et al. 2008, MNRAS, 399, 377

Marengo, M., Megeath, S. T., Fazio, G. G., et al. 2009, ApJ, 647, 1437

Metcalfe, T. S., Buccino, A. P., Brown, B. P., et al. 2013, ApJL, 763, L26

Minton, D. A., \& Malhotra, R. 2010, Icar, 207, 744

Müller, S., Löhne, T., \& Krivov, A. V. 2010, ApJ, 708, 1728

Murray-Clay, R. A., \& Schlichting, H. E. 2011, ApJ, 730, 132

Pilbratt, G. L., Riedinger, J. R., Passvogel, T., et al. 2010, A\&A, 518, L1

Poglitsch, A., Waelkens, C., Geis, N., et al. 2010, A\&A, 518, L2

Reffert, S., \& Quirrenbach, A. 2011, A\&A, 527, A140

Reidemeister, M., Krivov, A. V., Stark, C. C., et al. 2011, A\&A, 527, A57

Sibthorpe, B., Vandenbussche, B., Greaves, J. S., et al. 2010, A\&A, 518, L130

Su, K. Y. L., Rieke, G. H., Malhotra, M., et al. 2013, ApJ, 763, 118

Walsh, K. J., Morbidelli, A., Raymond, S. N., OBrien, D. P., \& Mandell, A. M. 2011, Natur, 475, 206

Wyatt, M. C. 2008, ARA\&A, 46, 339

Wyatt, M. C., Clarke, C. J., \& Booth, M. 2011, CMDA, 111, 1

Wyatt, M. C., Dermott, S. F., Telesco, C. M., et al. 1999, ApJ, 527, 918 